\title{
Towards a Liouville theorem for continuous viscosity solutions to fully nonlinear elliptic equations in conformal geometry
}

\author{
YanYan $\mathrm{Li}^{* \dagger}$ and Luc Nguyen ${ }^{\ddagger}$ and Bo Wang $\S$ \\ Dedicated to Gang Tian on his 60th birthday with friendship.
}

\begin{abstract}
We study entire continuous viscosity solutions to fully nonlinear elliptic equations involving the conformal Hessian. We prove the strong comparison principle and Hopf Lemma for (non-uniformly) elliptic equations when one of the competitors is $C^{1,1}$. We obtain as a consequence a Liouville theorem for entire solutions which are approximable by $C^{1,1}$ solutions on larger and larger compact domains, and, in particular, for entire $C_{\text {loc }}^{1,1}$ solutions: they are either constants or standard bubbles.
\end{abstract}

\section{Contents}

1 Introduction

2 The strong comparison principle and the Hopf Lemma

2.1 Proof of the strong comparison principle . . . . . . . . . . . 10

2.2 Proof of the Hopf Lemma . . . . . . . . . . . . . 20

*Department of Mathematics, Rutgers University, Hill Center, Busch Campus, 110 Frelinghuysen Road, Piscataway, NJ 08854, USA. Email: yyli@math.rutgers.edu.

${ }^{\dagger}$ Partially supported by NSF Grant DMS-1501004.

${ }^{\ddagger}$ Mathematical Institute and St Edmund Hall, University of Oxford, Andrew Wiles Building, Radcliffe Observatory Quarter, Woodstock Road, Oxford OX2 6GG, UK. Email: luc.nguyen@maths.ox.ac.uk.

${ }^{\S}$ School of Mathematics and Statistics, Beijing Institute of Technology, Beijing 100081, China. Email: wangbo89630@bit.edu.cn.

\Partially supported by NNSF (11701027) and Beijing Institute of Technology Research Fund Program for Young Scholars. 


\section{Introduction}

It is of interest to prove Liouville theorems for entire continuous viscosity solutions of a fully nonlinear elliptic equation of the form

$$
f\left(\lambda\left(A^{u}\right)\right)=1, \quad \lambda\left(A^{u}\right) \in \Gamma, \quad u>0 \text { on } \mathbb{R}^{n},
$$

where the conformal Hessian $A^{u}$ of $u$ is defined for $n \geq 3$ by

$$
A^{u}=-\frac{2}{n-2} u^{-\frac{n+2}{n-2}} \nabla^{2} u+\frac{2 n}{(n-2)^{2}} u^{-\frac{2 n}{n-2}} \nabla u \otimes \nabla u-\frac{2}{(n-2)^{2}} u^{-\frac{2 n}{n-2}}|\nabla u|^{2} I,
$$

$I$ is the $n \times n$ identity matrix, $\lambda\left(A^{u}\right)$ denotes the eigenvalues of $A^{u}, \Gamma$ is an open subset of $\mathbb{R}^{n}$ and $f \in C^{0}(\bar{\Gamma})$. (See [30], or Definition 2.2 below with $\psi=-\ln u$, for the definition of viscosity solutions as well as sub- and super-solutions.) Typically, $(f, \Gamma)$ is assumed to satisfy the following structural conditions.

(i) $(f, \Gamma)$ is symmetric, i.e.

$$
\text { if } \lambda \in \Gamma \text { and } \lambda^{\prime} \text { is a permutation of } \lambda \text {, then } \lambda^{\prime} \in \Gamma \text { and } f\left(\lambda^{\prime}\right)=f(\lambda) \text {. }
$$

(ii) $(f, \Gamma)$ is elliptic, i.e.

$$
\text { if } \lambda \in \Gamma \text { and } \mu \in \bar{\Gamma}_{n} \text {, then } \lambda+\mu \in \Gamma \text { and } f(\lambda+\mu) \geq f(\lambda),
$$

where $\Gamma_{n}:=\left\{\mu \in \mathbb{R}^{n}: \mu_{i}>0\right\}$ is the positive cone.

(iii) $(f, \Gamma)$ is locally strictly elliptic, i.e. for any compact subset $K$ of $\Gamma$, there is some constant $\delta(K)>0$ such that

$$
f(\lambda+\mu)-f(\lambda) \geq \delta(K)|\mu| \text { for all } \lambda \in K, \mu \in \bar{\Gamma}_{n} .
$$

(iv) $f$ is locally Lipschitz, i.e. for any compact subset $K$ of $\Gamma$, there is some constant $C(K)>0$ such that

$$
\left|f\left(\lambda^{\prime}\right)-f(\lambda)\right| \leq C(K)\left|\lambda^{\prime}-\lambda\right| \text { for all } \lambda, \lambda^{\prime} \in K
$$

(v) The 1-superlevel set of $f$ stays in $\Gamma$, namely

$$
f^{-1}([1, \infty)) \subset \Gamma \text {. }
$$


(vi) $\Gamma$ satisfies

$$
\Gamma \subset \Gamma_{1}:=\left\{\mu \in \mathbb{R}^{n}: \mu_{1}+\ldots \mu_{n}>0\right\}
$$

It should be noted that equation (11) is not necessarily uniformly elliptic and that we do not assume that $\Gamma$ be convex nor $f$ be concave.

Standard examples of $(f, \Gamma)$ satisfying (2)-(7) are given by $(f, \Gamma)=\left(\sigma_{k}^{1 / k}, \Gamma_{k}\right)$, $1 \leq k \leq n$, where $\sigma_{k}$ is the $k$-th elementary symmetric function and $\Gamma_{k}$ is the connected component of $\left\{\lambda \in \mathbb{R}^{n}: \sigma_{k}(\lambda)>0\right\}$ containing the positive cone $\Gamma_{n}$.

Liouville theorems for (11) have been studied extensively. We mention here earlier results of Gidas, Ni and Nirenberg [15], Caffarelli, Gidas and Spruck [10] in the semi-linear case, of Viaclovsky [39, 40] for the $\sigma_{k}$-equations for $C^{2}$ solutions which are regular at infinity, of Chang, Gursky and Yang [11] for the $\sigma_{2}$-equation in four dimensions, of $\mathrm{Li}$ and $\mathrm{Li}$ [26, 27] for $C^{2}$ solutions, and of $\mathrm{Li}$ and Nguyen [32] for continuous viscosity solutions which are approximable by $C^{2}$ solutions on larger and larger compact domains.

The key use of the $C^{2}$ regularity in the proof of the Liouville theorem in [32] is the strong comparison principle and Hopf Lemma for (1). In fact, if the strong comparison principle and Hopf Lemma can be established for $C^{1, \alpha}$ solutions $(0 \leq \alpha \leq 1)$, a Liouville theorem is then proved in $C^{1, \alpha}$ regularity by the same arguments.

The present note is an exploration in the above direction. We establish the strong comparison principle and Hopf Lemma when one competitor is $C^{1,1}$, and obtain as a consequence a Liouville theorem in this regularity.

Theorem 1.1 (Strong comparison principle). Let $\Omega$ be an open, connected subset of $\mathbb{R}^{n}, n \geq 3, \Gamma$ be a non-empty open subset of $\mathbb{R}^{n}$ and $f \in C^{0}(\bar{\Gamma})$ satisfying (2) -(6) . Assume that

(i) $u_{1} \in U S C(\Omega ;[0, \infty))$ and $u_{2} \in \operatorname{LSC}(\Omega ;(0, \infty])$ are a sub-solution and a supersolution to $f\left(\lambda\left(A^{u}\right)\right)=1$ in $\Omega$ in the viscosity sense, respectively,

(ii) and that $u_{1} \leq u_{2}$ in $\Omega$.

If one of $\ln u_{1}$ and $\ln u_{2}$ belongs to $C_{\text {loc }}^{1,1}(\Omega)$, then either $u_{1} \equiv u_{2}$ in $\Omega$ or $u_{1}<u_{2}$ in $\Omega$.

Theorem 1.2 (Hopf Lemma). Let $\Omega$ be an open subset of $\mathbb{R}^{n}, n \geq 3$, such that $\partial \Omega$ is $C^{2}$ near some point $\hat{x} \in \partial \Omega, \Gamma$ be a non-empty open subset of $\mathbb{R}^{n}$ and $f \in C^{0}(\bar{\Gamma})$ satisfying (2) -(6) . Assume that

(i) $u_{1} \in U S C(\Omega \cup\{\hat{x}\} ;[0, \infty))$ and $u_{2} \in L S C(\Omega \cup\{\hat{x}\} ;(0, \infty])$ are a sub-solution and a super-solution to $f\left(\lambda\left(A^{u}\right)\right)=1$ in $\Omega$ in the viscosity sense, respectively,

(ii) and that $u_{1}<u_{2}$ in $\Omega$, and $u_{1}(\hat{x})=u_{2}(\hat{x})$. 
If one of $\ln u_{1}$ and $\ln u_{2}$ belongs to $C^{1,1}(\Omega \cup\{\hat{x}\})$, then

$$
\liminf _{s \rightarrow 0^{+}} \frac{\left(u_{2}-u_{1}\right)(\hat{x}-s \nu(\hat{x}))}{s}>0,
$$

where $\nu(\hat{x})$ is the outward unit normal to $\partial \Omega$ at $\hat{x}$.

Our proof of the strong comparison principle and Hopf Lemma uses ideas in Caffarelli, Li and Nirenberg [9] and an earlier work of the authors [33]. In fact we establish them for more general equations of the form

$$
F\left(x, \psi, \nabla \psi, \nabla^{2} \psi\right)=1 .
$$

See Section 2, Theorem 2.3 and Theorem 2.4.

There has been a lot of studies on the (strong) comparison principle and Hopf Lemma for elliptic equations in related contexts. See for instance [1 9, 12, 14, 16 25 , 29 31, 33 36, 38, 41 and the references therein.

As mentioned earlier, a combination of the above strong comparison principle and Hopf Lemma and the proof of [32, Theorem 1.1] give the following Liouville theorem.

Theorem 1.3 (Liouville theorem). Assume that $n \geq 3$ and $(f, \Gamma)$ satisfies (22)-(7). Suppose that there exist $v_{k} \in C^{1,1}\left(B_{R_{k}}(0)\right), R_{k} \rightarrow \infty$, such that $f\left(\lambda\left(A^{v_{k}}\right)\right)=1$, $\lambda\left(A^{v_{k}}\right) \in \Gamma$ in the ball $B_{R_{k}}(0)$ of radius $R_{k}$ in the viscosity sense, $v_{k}$ converges uniformly on compact subsets of $\mathbb{R}^{n}$ to some function $v>0$. Then

either (i) $v$ is identically constant, $0 \in \Gamma$ and $f(0)=1$,

or (ii) $v$ has the form

$$
v(x)=\left(\frac{a}{1+b^{2}\left|x-x_{0}\right|^{2}}\right)^{\frac{n-2}{2}}
$$

for some $x_{0} \in \mathbb{R}^{n}$ and some $a, b>0$ satisfying $f\left(2 b^{2} a^{-2}, \ldots, 2 b^{2} a^{-2}\right)=1$.

It is a fact that if $u$ is $C^{1,1}$ in some open set $\Omega, u$ satisfies $f\left(\lambda\left(A^{u}\right)\right)=1$ in the viscosity sense in $\Omega$ if and only if it satisfies $f\left(\lambda\left(A^{u}\right)\right)=1$ almost everywhere in $\Omega$. See e.g. Lemma 2.5.

It should be clear that if $0 \in \Gamma$ and $f(0)=1$, then, by (3) and (4),$(t, \ldots, t) \in \Gamma$ and $f(t, \ldots, t)>1$ for all $t>0$. Hence if some constant is a solution of (1), then all entire solutions of (11) are constant, and likewise if some function of the form (8) is a solution of (11), then all entire solutions of (11) are of the form (8).

An immediate consequence is:

Corollary 1.4. Assume that $n \geq 3$ and $(f, \Gamma)$ satisfies (2)-(17). If $v \in C_{\mathrm{loc}}^{1,1}\left(\mathbb{R}^{n}\right)$ is a viscosity solution of (11), then $v$ is either a constant or of the form (8). 
The rest of the paper contains two sections. In Section 2, we state and prove our strong comparison principle and Hopf Lemma for a class of elliptic equations which is more generalized than $f\left(\lambda\left(A^{u}\right)\right)=1$. In Section 3 , we prove the Liouville theorem (Theorem 1.3).

\section{The strong comparison principle and the Hopf Lemma}

In this section we prove the strong comparison principle and the Hopf Lemma for elliptic equations of the form

$$
F\left(x, \psi, \nabla \psi, \nabla^{2} \psi\right)=1 \text { in } \Omega
$$

where $\Omega$ is an open subset of $\mathbb{R}^{n}, n \geq 1, F \in C(\overline{\mathscr{U}})$, $\mathscr{U}$ is a non-empty open subset of $\bar{\Omega} \times \mathbb{R} \times \mathbb{R}^{n} \times \operatorname{Sym}_{n}$, and $(F, \mathscr{U})$ satisfies the following conditions.

(i) $(F, \mathscr{U})$ is elliptic, i.e. for all $(x, s, p, M) \in \mathscr{U}, N \in \operatorname{Sym}_{n}, N \geq 0$,

$$
(x, s, p, M+N) \in \mathscr{U} \text { and } F(x, s, p, M+N) \geq F(x, s, p, M) .
$$

Here and below we write $N \geq 0$ for a non-negative definite matrix $N$.

(ii) For $x \in \bar{\Omega}$, let $\mathscr{U}_{x}:=\left\{(s, p, M) \in \mathbb{R} \times \mathbb{R}^{n} \times \operatorname{Sym}_{n}:(x, s, p, M) \in \mathscr{U}\right\}$. Then, for $x \in \bar{\Omega}$, the 1 -superlevel set of $F(x, \cdot)$ stays in $\mathscr{U}_{x}$, i.e.

$$
F(x, s, p, M)<1 \text { for all } x \in \bar{\Omega} \text { and }(s, p, M) \in \partial \mathscr{U}_{x},
$$

or, equivalently,

$$
\left\{(s, p, M) \in \overline{\mathscr{U}}_{x}: F(x, s, p, M) \geq 1\right\} \subset \mathscr{U}_{x}
$$

(iii) $(F, \mathscr{U})$ is locally strictly elliptic, i.e. for any compact subset $\mathscr{K}$ of $\mathscr{U}$, there is some constant $\delta=\delta(\mathscr{K})>0$ such that, for all $(x, s, p, M) \in \mathscr{K}, N \in$ $\mathrm{Sym}_{n}, N \geq 0$,

$$
F(x, s, p, M+N)-F(x, s, p, M) \geq \delta(\mathscr{K})|N|
$$

(iv) $F$ satisfies a local Lipschitz condition with respect to $(s, p, M)$, namely for every compact subset $\mathscr{K}$ of $\mathscr{U}$, there there exists $C(\mathscr{K})>0$ such that, for all $(x, s, p, M),\left(x, s^{\prime}, p^{\prime}, M^{\prime}\right) \in \mathscr{K}$,

$$
\left|F(x, s, p, M)-F\left(x, s^{\prime}, p^{\prime}, M^{\prime}\right)\right| \leq C(\mathscr{K})\left(\left|s-s^{\prime}\right|+\left|p-p^{\prime}\right|+\left|M-M^{\prime}\right|\right) .
$$


To keep the notation compact, we abbreviate

$$
J_{2}[\psi]=\left(\psi, \nabla \psi, \nabla^{2} \psi\right) \in \mathbb{R} \times \mathbb{R}^{n} \times \operatorname{Sym}_{n}
$$

We note that equation (11) can be put in the form (9) by writing $\psi=-\ln u$, $F\left(J_{2}[\psi]\right)=f\left(\lambda\left(A^{u}\right)\right)$.

To dispel confusion, we remark that $\mathscr{U}$ is defined as a subset of $\bar{\Omega} \times \mathbb{R} \times \mathbb{R}^{n} \times \mathrm{Sym}_{n}$ rather than that of $\Omega \times \mathbb{R} \times \mathbb{R}^{n} \times \mathrm{Sym}_{n}$. In particular, the 'local' properties in (iii)-(iv) are local with respect to the $(s, p, M)$-variables and not the $x$-variables.

Let us start with the definition of classical and viscosity (sub-/super-)solutions. For this we only need the ellipticity condition (10) and the following condition which is weaker than (11):

(ii') There holds

$$
F(x, s, p, M) \leq 1 \text { for all } x \in \bar{\Omega} \text { and }(s, p, M) \in \partial \mathscr{U}_{x}
$$

or, equivalently,

$$
\left\{(s, p, M) \in \overline{\mathscr{U}}_{x}: F(x, s, p, M)>1\right\} \subset \mathscr{U}_{x} .
$$

Definition 2.1 (Classical (sub-/super-)solutions). Let $\Omega \subset \mathbb{R}^{n}, n \geq 1$, be an open set, and $\mathscr{U}$ be a non-empty open subset of $\bar{\Omega} \times \mathbb{R} \times \mathbb{R}^{n} \times \operatorname{Sym}_{n}$ and $F \in C^{0}(\overline{\mathscr{U}})$ satisfying (10) and (14). For a function $\psi \in C^{2}(\Omega)$, we say that

$$
F\left(x, J_{2}[\psi]\right) \leq 1 \quad\left(F\left(x, J_{2}[\psi]\right) \geq 1 \text { resp. }\right) \quad \text { classically in } \Omega
$$

if there holds

$$
\begin{gathered}
\text { either }\left(x, J_{2}[\psi](x)\right) \notin \overline{\mathscr{U}} \text { or } F\left(x, J_{2}[\psi](x)\right) \leq 1 \text { for all } x \in \Omega \\
\left(\left(x, J_{2}[\psi](x)\right) \in \overline{\mathscr{U}} \text { and } F\left(x, J_{2}[\psi](x)\right) \geq 1 \text { for all } x \in \Omega \text { resp. }\right) .
\end{gathered}
$$

We say that a function $\psi \in C^{2}(\Omega)$ is a classical solution of (9) in $\Omega$ if we have that $\left(x, J_{2}[\psi](x)\right) \in \overline{\mathscr{U}}$ and $F\left(x, J_{2}[\psi](x)\right)=1$ for every $x \in \Omega$.

When $F\left(x, J_{2}[\psi]\right) \leq 1\left(F\left(x, J_{2}[\psi]\right) \geq 1\right.$, resp. $)$ in $\Omega$, we also say interchangeably that $u$ is a super-solution (sub-solution) to (9) in $\Omega$.

In the above definition, the role of condition (14) is manifested in the property that if $\psi_{k}$ is a sequence of super-solutions which converges in $C^{2}$ to some $\psi$, then $\psi$ is also a super-solution. When discussing only sub-solutions, condition (14) can be dropped. 
Definition 2.2 (Viscosity (sub-/super-)solutions). Let $\Omega \subset \mathbb{R}^{n}, n \geq 1$, be an open set, and $\mathscr{U}$ be a non-empty open subset of $\bar{\Omega} \times \mathbb{R} \times \mathbb{R}^{n} \times \operatorname{Sym}_{n}$ and $F \in C^{0}(\overline{\mathscr{U}})$ satisfying (10) and (14). For a function $\psi \in \operatorname{LSC}(\Omega ; \mathbb{R} \cup\{\infty\})(\psi \in U S C(\Omega ; \mathbb{R} \cup\{-\infty\})$ resp.), we say that

$$
F\left(x, J_{2}[\psi]\right) \leq 1 \quad\left(F\left(x, J_{2}[\psi]\right) \geq 1 \text { resp. }\right) \quad \text { in } \Omega
$$

in the viscosity sense if for any $x_{0} \in \Omega, \varphi \in C^{2}(\Omega),(\psi-\varphi)\left(x_{0}\right)=0$ and

$$
\psi-\varphi \geq 0 \quad(\psi-\varphi \leq 0 \text { resp. }) \quad \text { near } x_{0}
$$

there holds

$$
\begin{gathered}
\text { either }\left(x_{0}, J_{2}[\varphi]\left(x_{0}\right)\right) \notin \overline{\mathscr{U}} \text { or } F\left(x_{0}, J_{2}[\varphi]\left(x_{0}\right)\right) \leq 1 \\
\left(\left(x_{0}, J_{2}[\varphi]\left(x_{0}\right)\right) \in \overline{\mathscr{U}} \text { and } F\left(x_{0}, J_{2}[\varphi]\left(x_{0}\right)\right) \geq 1 \text { resp. }\right) .
\end{gathered}
$$

We say that a function $\psi \in C^{0}(\Omega)$ satisfies (9) in the viscosity sense in $\Omega$ if we have both that $F\left(x, J_{2}[\psi]\right) \geq 1$ and $F\left(x, J_{2}[\psi]\right) \leq 1$ in $\Omega$ in the viscosity sense.

When $F\left(x, J_{2}[\psi]\right) \leq 1\left(F\left(x, J_{2}[\psi]\right) \geq 1\right.$, resp. $)$ in $\Omega$ in the viscosity sense, we also say interchangeably that $u$ is a viscosity super-solution (sub-solution) to (9) in $\Omega$.

The main results in this section are the following.

Theorem 2.3 (Strong comparison principle). Let $\Omega$ be an open, connected subset of $\mathbb{R}^{n}, n \geq 1$, $\mathscr{U}$ be a non-empty open subset of $\bar{\Omega} \times \mathbb{R} \times \mathbb{R}^{n} \times \operatorname{Sym}_{n}$ and $F \in C^{0}(\overline{\mathscr{U}})$ satisfying (10)-(13). Assume that

(i) $\psi_{1} \in U S C(\Omega ; \mathbb{R} \cup\{-\infty\})$ and $\psi_{2} \in L S C(\Omega ; \mathbb{R} \cup\{\infty\})$ are a sub-solution and a super-solution to (9) in $\Omega$ in the viscosity sense, respectively,

(ii) and that $\psi_{1} \leq \psi_{2}$ in $\Omega$.

If one of $\psi_{1}$ and $\psi_{2}$ belongs to $C_{\mathrm{loc}}^{1,1}(\Omega)$, then either $\psi_{1} \equiv \psi_{2}$ in $\Omega$ or $\psi_{1}<\psi_{2}$ in $\Omega$.

Theorem 2.4 (Hopf Lemma). Let $\Omega$ be an open subset of $\mathbb{R}^{n}, n \geq 1$, such that $\partial \Omega$ is $C^{2}$ near some point $\hat{x} \in \partial \Omega, \mathscr{U}$ be a non-empty open subset of $\bar{\Omega} \times \mathbb{R} \times \mathbb{R}^{n} \times \mathrm{Sym}_{n}$ and $F \in C^{0}(\overline{\mathscr{U}})$ satisfying (10) $-(13)$. A ssume that

(i) $\psi_{1} \in U S C(\Omega \cup\{\hat{x}\} ; \mathbb{R} \cup\{-\infty\})$ and $\psi_{2} \in L S C(\Omega \cup\{\hat{x}\} ; \mathbb{R} \cup\{\infty\})$ are a subsolution and a super-solution to (9) in $\Omega$ in the viscosity sense, respectively,

(ii) and that $\psi_{1}<\psi_{2}$ in $\Omega$, and $\psi_{1}(\hat{x})=\psi_{2}(\hat{x})$.

If one of $\psi_{1}$ and $\psi_{2}$ belongs to $C^{1,1}(\Omega \cup\{\hat{x}\})$, then

$$
\liminf _{s \rightarrow 0^{+}} \frac{\left(\psi_{2}-\psi_{1}\right)(\hat{x}-s \nu(\hat{x}))}{s}>0
$$

where $\nu(\hat{x})$ is the outward unit normal to $\partial \Omega$ at $\hat{x}$. 
If $\psi_{1}$ and $\psi_{2}$ are continuous and one of them is $C^{2}$, the above theorems were proved in Caffarelli, Li, Nirenberg [9].

Before turning to the proof of the above theorems, we give some simple statements for viscosity solutions.

Lemma 2.5. Let $\Omega \subset \mathbb{R}^{n}, n \geq 1$, be an open set, and $\mathscr{U}$ be a non-empty open subset of $\bar{\Omega} \times \mathbb{R} \times \mathbb{R}^{n} \times \mathrm{Sym}_{n}$ and $F \in C^{0}(\overline{\mathscr{U}})$ satisfying (10) and (14). Suppose that $\psi$ is semi-concave (semi-convex resp.) in $\Omega$, then

$$
F\left(x, J_{2}[\psi]\right) \leq 1 \quad\left(F\left(x, J_{2}[\psi]\right) \geq 1 \text { resp. }\right) \quad \text { in } \Omega \text { in the viscosity sense }
$$

if and only if

$$
\begin{gathered}
\text { either }\left(x, J_{2}[\psi](x)\right) \notin \overline{\mathscr{U}} \text { or } F\left(x, J_{2}[\psi](x)\right) \leq 1 \quad \text { a.e. in } \Omega \\
\left(\left(x, J_{2}[\psi](x)\right) \in \overline{\mathscr{U}} \text { and } F\left(x, J_{2}[\psi](x)\right) \geq 1 \quad \text { a.e. in } \Omega \text { resp. }\right) .
\end{gathered}
$$

Recall that $\psi$ is semi-concave (semi-convex resp.) in $\Omega$ if there is some $K>0$ such that $\psi-\frac{K}{2}|x|^{2}\left(\psi+\frac{K}{2}|x|^{2}\right.$ resp.) is locally concave (convex resp.) in $\Omega$. By a theorem of Alexandrov, Buselman and Feller (see e.g. [8, Theorem 1.5]), semi-concave (or semi-convex) functions are almost everywhere punctually second order differentiable.

Proof. (a) Consider the inequality $F\left(x, J_{2}[\psi]\right) \leq 1$.

Since $\psi$ is semi-concave, it is almost everywhere punctually second order differentiable. Suppose that $F\left(x, J_{2}[\psi]\right) \leq 1$ in $\Omega$ in the viscosity sense and $x_{0}$ is a point where $\psi$ is punctually second order differentiable. Then we can use

$$
\varphi(x)=\psi\left(x_{0}\right)+\nabla \psi\left(x_{0}\right) \cdot\left(x-x_{0}\right)+\left(x-x_{0}\right)^{T} \nabla^{2} \psi\left(x_{0}\right)\left(x-x_{0}\right)-\delta\left|x-x_{0}\right|^{2}
$$

for any $\delta>0$ as test functions at $x_{0}$ to see that

$$
\text { either }\left(x_{0}, J_{2}[\psi]\left(x_{0}\right)-(0,0,2 \delta I)\right) \notin \overline{\mathscr{U}} \text { or } F\left(x_{0}, J_{2}[\psi]\left(x_{0}\right)-(0,0,2 \delta I)\right) \leq 1 \text {. }
$$

Sending $\delta \rightarrow 0$ and using (14), we obtain

$$
\text { either }\left(x_{0}, J_{2}[\psi]\left(x_{0}\right)\right) \notin \overline{\mathscr{U}} \text { or } F\left(x_{0}, J_{2}[\psi]\left(x_{0}\right)\right) \leq 1 \text {. }
$$

Conversely, assume that either $\left(x, J_{2}[\psi](x)\right) \notin \overline{\mathscr{U}}$ or $F\left(x, J_{2}[\psi](x)\right) \leq 1$ for almost all $x \in \Omega$, and suppose, for some $x_{0} \in \Omega$ and $\varphi \in C^{2}(\Omega)$, that $(\psi-\varphi)\left(x_{0}\right)=0$ and $\psi-\varphi \geq 0$ near $x_{0}$. We need to show that

$$
\text { either }\left(x_{0}, J_{2}[\varphi]\left(x_{0}\right)\right) \notin \overline{\mathscr{U}} \text { or } F\left(x_{0}, J_{2}[\varphi]\left(x_{0}\right)\right) \leq 1 \text {. }
$$


If $\left(x_{0}, J_{2}[\varphi]\left(x_{0}\right)\right) \notin \mathscr{U}$, we are done by (14). We assume henceforth that $\left(x_{0}, J_{2}[\varphi]\left(x_{0}\right)\right) \in$ $\mathscr{U}$.

Replacing $\varphi$ by $\varphi-\delta\left|x-x_{0}\right|^{2}$ for some small $\delta>0$ and letting $\delta \rightarrow 0$ eventually, we may assume without loss of generality that

$$
\psi>\varphi \text { in } B_{2 r_{0}}\left(x_{0}\right) \backslash\left\{x_{0}\right\} \subset \Omega \text { for some } r_{0}>0 .
$$

For small $\eta>0$, let $\xi=\xi_{\eta}=(\psi-\varphi-\eta)^{-}$and let $\Gamma_{\xi}$ be the concave envelop of $\xi$ in $B_{2 r_{0}}\left(x_{0}\right)$. We have by [8, Lemma 3.5] that

$$
\int_{\left\{\xi=\Gamma_{\xi}\right\}} \operatorname{det}\left(-\nabla^{2} \Gamma_{\xi}\right) \geq \frac{1}{C}\left(\sup _{B_{2 r_{0}}\left(x_{0}\right)} \xi\right)^{n}>0 .
$$

In particular, the set $\left\{\xi=\Gamma_{\xi}\right\}$ has non-zero measure. Thus, we can find $y_{\eta} \in\left\{\xi=\Gamma_{\xi}\right\}$ such that $\psi$ is punctually second order differentiable at $y_{\eta}$, either $\left(y_{\eta}, J_{2}[\psi]\left(y_{\eta}\right)\right) \notin \overline{\mathscr{U}}$ or $F\left(y_{\eta}, J_{2}[\psi]\left(y_{\eta}\right)\right) \leq 1$ and

$$
\begin{aligned}
0>\xi\left(y_{\eta}\right) & =\psi\left(y_{\eta}\right)-\varphi\left(y_{\eta}\right)-\eta \geq-\eta, \\
\left|\nabla \xi\left(y_{\eta}\right)\right| & =\left|\nabla \psi\left(y_{\eta}\right)-\nabla \varphi\left(y_{\eta}\right)\right| \leq C \eta, \\
\nabla^{2} \xi\left(y_{\eta}\right) & =\nabla^{2} \psi\left(y_{\eta}\right)-\nabla^{2} \varphi\left(y_{\eta}\right) \geq 0 .
\end{aligned}
$$

Recalling that $\left(x_{0}, J_{2}[\varphi]\left(x_{0}\right)\right) \in \mathscr{U}$ and noting that $y_{\eta} \rightarrow x_{0}$ as $\eta \rightarrow 0$, we deduce from (10) and (15)-(17) that, for all small $\eta,\left(y_{\eta}, J_{2}[\varphi]\left(y_{\eta}\right)\right),\left(y_{\eta}, J_{2}[\psi]\left(y_{\eta}\right)\right)$ and $\left(y_{\eta}, \psi\left(y_{\eta}\right), \nabla \psi\left(y_{\eta}\right), \nabla^{2} \varphi\left(y_{\eta}\right)\right)$ belong to $\mathscr{U}$. We then have

$$
\begin{array}{ll}
1 \geq & F\left(y_{\eta}, J_{2}[\psi]\left(y_{\eta}\right)\right) \\
\stackrel{(10),(17)}{\geq} & F\left(y_{\eta}, \psi\left(y_{\eta}\right), \nabla \psi\left(y_{\eta}\right), \nabla^{2} \varphi\left(y_{\eta}\right)\right) \\
\stackrel{(15,,(16)}{\geq} & F\left(y_{\eta}, \varphi\left(y_{\eta}\right), \nabla \varphi\left(y_{\eta}\right), \nabla^{2} \varphi\left(y_{\eta}\right)\right)+o_{\eta}(1),
\end{array}
$$

where $o_{\eta}(1) \rightarrow 0$ as $\eta \rightarrow 0$ and where we have used the uniform continuity of $F$ on compact subsets of $\overline{\mathscr{U}}$. Letting $\eta \rightarrow 0$, we obtain the assertion.

(b) Consider now the inequality $F\left(x, J_{2}[\psi]\right) \geq 1$. This case is treated similarly, but is slightly easier as we do not have a dichotomy in the almost everywhere sense.

Since $\psi$ is semi-convex, it is almost everywhere punctually second order differentiable. If $F\left(x, J_{2}[\psi]\right) \geq 1$ is satisfied in the viscosity sense, then, as in the previous case, if $x_{0}$ is a point where $\psi$ is punctually second order differentiable, then

$$
\left(x_{0}, J_{2}[\psi]\left(x_{0}\right)+(0,0,2 \delta)\right) \in \overline{\mathscr{U}} \text { and } F\left(x_{0}, J_{2}[\psi]\left(x_{0}\right)+(0,0,2 \delta)\right) \geq 1 \text { for any } \delta>0,
$$

and so, upon sending $\delta \rightarrow 0$, we obtain

$$
\left(x_{0}, J_{2}[\psi]\left(x_{0}\right)\right) \in \overline{\mathscr{U}} \text { and } F\left(x_{0}, J_{2}[\psi]\left(x_{0}\right)\right) \geq 1 \text {. }
$$


Suppose that $F\left(x, J_{2}[\psi](x)\right) \geq 1$ holds almost everywhere in $\Omega$ and suppose, for some $x_{0} \in \Omega$ and $\varphi \in C^{2}(\Omega)$, that $(\psi-\varphi)\left(x_{0}\right)=0$ and $\psi-\varphi \leq 0$ near $x_{0}$. We need to show that

$$
F\left(x_{0}, J_{2}[\varphi]\left(x_{0}\right)\right) \geq 1 .
$$

Replacing $\varphi$ by $\varphi+\delta\left|x-x_{0}\right|^{2}$ for some small $\delta>0$ and letting $\delta \rightarrow 0$ eventually, we may assume without loss of generality that

$$
\psi<\varphi \text { in } B_{2 r_{0}}\left(x_{0}\right) \backslash\left\{x_{0}\right\} \subset \Omega \text { for some } r_{0}>0 \text {. }
$$

For small $\eta>0$, let $\xi=\xi_{\eta}=(\psi-\varphi+\eta)^{+}$and let $\Gamma_{\xi}$ be the concave envelop of $\xi$ in $B_{2 r_{0}}\left(x_{0}\right)$. We have by [8, Lemma 3.5] that

$$
\int_{\left\{\xi=\Gamma_{\xi}\right\}} \operatorname{det}\left(-\nabla^{2} \Gamma_{\xi}\right) \geq \frac{1}{C}\left(\sup _{B_{2 r_{0}}\left(x_{0}\right)} \xi\right)^{n}>0 .
$$

In particular, the set $\left\{\xi=\Gamma_{\xi}\right\}$ has positive measure. Thus, we can find $y_{\eta} \in\left\{\xi=\Gamma_{\xi}\right\}$ such that $\psi$ is punctually second order differentiable at $y_{\eta}, F\left(y_{\eta}, J_{2}[\psi]\left(y_{\eta}\right)\right) \geq 1$ and

$$
\begin{aligned}
0<\xi\left(y_{\eta}\right) & =\psi\left(y_{\eta}\right)-\varphi\left(y_{\eta}\right)+\eta \leq \eta \\
\left|\nabla \xi\left(y_{\eta}\right)\right| & =\left|\nabla \psi\left(y_{\eta}\right)-\nabla \varphi\left(y_{\eta}\right)\right| \leq C \eta \\
\nabla^{2} \xi\left(y_{\eta}\right) & =\nabla^{2} \psi\left(y_{\eta}\right)-\nabla^{2} \varphi\left(y_{\eta}\right) \leq 0 .
\end{aligned}
$$

It follows that

$$
\begin{aligned}
1 \leq & F\left(y_{\eta}, J_{2}[\psi]\left(y_{\eta}\right)\right) \\
\stackrel{(10),(20)}{\leq} & F\left(y_{\eta}, \psi\left(y_{\eta}\right), \nabla \psi\left(y_{\eta}\right), \nabla^{2} \varphi\left(y_{\eta}\right)\right) \\
\stackrel{187,(19)}{\leq} & F\left(y_{\eta}, \varphi\left(y_{\eta}\right), \nabla \varphi\left(y_{\eta}\right), \nabla^{2} \varphi\left(y_{\eta}\right)\right)+o_{\eta}(1),
\end{aligned}
$$

where $o_{\eta}(1) \rightarrow 0$ as $\eta \rightarrow 0$ and where we have used the uniform continuity of $F$ on compact subsets of $\overline{\mathscr{U}}$. Letting $\eta \rightarrow 0$ and noting that $y_{\eta} \rightarrow x_{0}$, we conclude the proof.

\subsection{Proof of the strong comparison principle}

We first prove the strong comparison principle for subsolutions and $C^{1,1}$ strict supersolutions.

Proposition 2.6. Let $\Omega$ be an open, connected subset of $\mathbb{R}^{n}, n \geq 1$, $\mathscr{U}$ be a nonempty open subset of $\bar{\Omega} \times \mathbb{R} \times \mathbb{R}^{n} \times \operatorname{Sym}_{n}$ and $F \in C^{0}(\overline{\mathscr{U}})$ satisfying (10)-(11). Assume that 
(i) $\psi_{1} \in U S C(\Omega ; \mathbb{R} \cup\{-\infty\})$ satisfies

$$
F\left(x, J_{2}\left[\psi_{1}\right]\right) \geq 1 \text { in } \Omega \text { in the viscosity sense, }
$$

(ii) $\psi_{2} \in C_{\mathrm{loc}}^{1,1}(\Omega)$ satisfies for some constant $a<1$,

$$
\text { either }\left(x, J_{2}\left[\psi_{2}\right](x)\right) \notin \overline{\mathscr{U}} \text { or } F\left(x, J_{2}\left[\psi_{2}\right](x)\right) \leq a \quad \text { a.e. in } \Omega \text {, }
$$

(iii) $\psi_{1} \leq \psi_{2}$ in $\Omega$ and $\psi_{1}<\psi_{2}$ near $\partial \Omega$.

Then $\psi_{1}<\psi_{2}$ in $\Omega$.

Proof. We follow [33]. Assume by contradiction that there exists some $\hat{x} \in \Omega$ such that $\psi_{1}(\hat{x})=\psi_{2}(\hat{x})$.

Step 1: We regularize $\psi_{1}$ using sup-convolution.

This step is well known, see e.g. [8, Chapter 5].

Take some bounded domain $A$ containing $\hat{x}$ such that $\bar{A} \subset \Omega$ and $\psi_{1}<\psi_{2}$ on $\partial A$.

We define, for small $\varepsilon>0$ and $x \in A$,

$$
\hat{\psi}_{\varepsilon}(x)=\sup _{y \in \Omega}\left(\psi_{1}(y)-\frac{1}{\varepsilon}|x-y|^{2}\right) .
$$

It is well-known that $\hat{\psi}_{\varepsilon} \geq \psi_{1}, \hat{\psi}_{\varepsilon}$ is semi-convex, $\nabla^{2} \hat{\psi}_{\varepsilon} \geq-\frac{2}{\varepsilon} I$ a.e. in $A$, and $\hat{\psi}_{\varepsilon}$ converges monotonically to $\psi_{1}$ as $\varepsilon \rightarrow 0$. Furthermore, for every $x \in A$, there exists $x^{*}=x^{*}(\varepsilon, x)$ such that

$$
\hat{\psi}_{\varepsilon}(x)=\psi_{1}\left(x^{*}\right)-\frac{1}{\varepsilon}\left|x-x^{*}\right|^{2} .
$$

We note that if $x$ is a point where $\hat{\psi}_{\varepsilon}$ is punctually second order differentiable, then $\psi_{1}$ 'can be touched from above' at $x^{*}$ by a quadratic polynomial:

$$
\psi_{1}\left(x^{*}+z\right) \leq \hat{\psi}_{\varepsilon}(x)+\frac{1}{\varepsilon}\left|x^{*}-x\right|^{2}+\nabla \hat{\psi}_{\varepsilon}(x) \cdot z+\frac{1}{2} z^{T} \nabla^{2} \hat{\psi}_{\varepsilon}(x) z+o\left(|z|^{2}\right) \quad \text { as } z \rightarrow 0,
$$

which is a consequence of the inequalities

$$
\begin{aligned}
& \hat{\psi}_{\varepsilon}(x+z) \leq \hat{\psi}_{\varepsilon}(x)+\nabla \hat{\psi}_{\varepsilon}(x) \cdot z+\frac{1}{2} z^{T} \nabla^{2} \hat{\psi}_{\varepsilon}(x) z+o\left(|z|^{2}\right), \quad \text { as } z \rightarrow 0, \\
& \hat{\psi}_{\varepsilon}(x+z) \geq \psi_{1}\left(x^{*}+z\right)-\frac{1}{\varepsilon}\left|x^{*}-x\right|^{2} .
\end{aligned}
$$

(Here we have used the definition of $\hat{\psi}_{\varepsilon}$ in the last inequality.) 
An immediate consequence of (21)-(22) and the fact that $\psi_{1}$ is a sub-solution of (9) is that

$$
F\left(x^{*}, \hat{\psi}_{\varepsilon}(x)+\frac{1}{\varepsilon}\left|x^{*}-x\right|^{2}, \nabla \hat{\psi}_{\varepsilon}(x), \nabla^{2} \hat{\psi}_{\varepsilon}(x)\right) \geq 1 .
$$

Step 2: We proceed to derive a contradiction as in [33].

For small $\eta>0$, let $\tau=\tau(\varepsilon, \eta)$ be such that

$$
\eta=\sup _{A}\left(\hat{\psi}_{\varepsilon}-\psi_{2}+\tau\right) \text {. }
$$

Then

$$
\begin{aligned}
& \tau=\psi_{1}(\hat{x})-\psi_{2}(\hat{x})+\tau \leq \hat{\psi}_{\varepsilon}(\hat{x})-\psi_{2}(\hat{x})+\tau \leq \eta \\
& \tau=\eta-\sup _{A}\left(\hat{\psi}_{\varepsilon}-\psi_{2}\right) \geq \eta-\sup _{A}\left(\hat{\psi}_{\varepsilon}-\psi_{1}\right) .
\end{aligned}
$$

Suppose that $\varepsilon$ and $\eta$ are sufficiently small so that $\xi:=\hat{\psi}_{\varepsilon}-\psi_{2}+\tau$ is negative on $\partial A$. Let $\Gamma_{\xi^{+}}$denote the concave envelop of $\xi^{+}=\max (\xi, 0)$. Since $\xi$ is semi-convex and $\xi \leq 0$ on $\partial A$, we have by [8, Lemma 3.5] that

$$
\int_{\left\{\xi=\Gamma_{\xi^{+}}\right\}} \operatorname{det}\left(-\nabla^{2} \Gamma_{\xi^{+}}\right) \geq \frac{1}{C(\Omega)}\left(\sup _{\Omega} \xi\right)^{n}>0 .
$$

In particular, the set $\left\{\xi=\Gamma_{\xi^{+}}\right\}$has positive measure. Recall that $\hat{\psi}_{\varepsilon}$ and $\psi_{2}$ is almost everywhere punctually second order differentiable, we can find $y=y_{\varepsilon, \eta} \in\left\{\xi=\Gamma_{\xi^{+}}\right\}$ such that $\hat{\psi}_{\varepsilon}$ and $\psi_{2}$ are punctually second order differentiable at $y,\left|J_{2}\left[\psi_{2}\right](y)\right| \leq$ $C\|\psi\|_{C^{1,1}(\bar{A})}$, either $\left(y, J_{2}\left[\psi_{2}\right](y)\right) \notin \overline{\mathscr{U}}$ or $F\left(y, J_{2}\left[\psi_{2}\right](y)\right) \leq a$, and

$$
\begin{gathered}
0<\xi(y)=\hat{\psi}_{\varepsilon}(y)-\psi_{2}(y)+\tau \leq \eta, \\
|\nabla \xi(y)|=\left|\nabla \hat{\psi}_{\varepsilon}(y)-\nabla \psi_{2}(y)\right| \leq C \eta, \\
\nabla^{2} \xi(y)=\nabla^{2} \hat{\psi}_{\varepsilon}(y)-\nabla^{2} \psi_{2}(y) \leq 0 .
\end{gathered}
$$

We claim that

$$
\liminf _{\varepsilon \rightarrow 0} \frac{1}{\varepsilon}\left|y^{*}-y\right|^{2} \leq \eta,
$$

where $y^{*}=x^{*}(\varepsilon, y)$ and $x^{*}$ is defined in (21).

Let us assume (29) for now and go on with the proof. From, (10), (23), (28), we have $\left(y^{*}, \hat{\psi}_{\varepsilon}(y)+\frac{1}{\varepsilon}\left|y^{*}-y\right|^{2}, \nabla \hat{\psi}_{\varepsilon}(y), \nabla^{2} \psi_{2}(y)\right) \in \overline{\mathscr{U}}$ and

$$
\begin{aligned}
& 1 \stackrel{\sqrt[23]{\leq}}{\leq} F\left(y^{*}, \hat{\psi}_{\varepsilon}(y)+\frac{1}{\varepsilon}\left|y^{*}-y\right|^{2}, \nabla \hat{\psi}_{\varepsilon}(y), \nabla^{2} \hat{\psi}_{\varepsilon}(y)\right) \\
& \stackrel{\text { (10), (28) }}{\leq} F\left(y^{*}, \hat{\psi}_{\varepsilon}(y)+\frac{1}{\varepsilon}\left|y^{*}-y\right|^{2}, \nabla \hat{\psi}_{\varepsilon}(y), \nabla^{2} \psi_{2}(y)\right) \text {. }
\end{aligned}
$$


By the boundedness of $J_{2}\left[\psi_{2}\right](y)$, we may assume that

$$
\left(y, J_{2}\left[\psi_{2}\right](y)\right)=\left(y_{\varepsilon, \eta}, J_{2}\left[\psi_{2}\right]\left(y_{\varepsilon, \eta}\right)\right) \rightarrow\left(y_{0}, p_{0}\right) \text { along a sequence } \varepsilon, \eta \rightarrow 0 \text {. }
$$

By (24), (25), (26) and (27), we then have

$$
\left(y^{*}, \hat{\psi}_{\varepsilon}(y)+\frac{1}{\varepsilon}\left|y^{*}-y\right|^{2}, \nabla \hat{\psi}_{\varepsilon}(y), \nabla^{2} \psi_{2}(y)\right) \rightarrow\left(y_{0}, p_{0}\right) .
$$

Thus by (11) and (30), $\left(y_{0}, p_{0}\right) \in \mathscr{U}$ and $F\left(y_{0}, p_{0}\right) \geq 1$. But this implies, in view of (31), that $\left(y, J_{2}\left[\psi_{2}\right](y)\right) \in \mathscr{U}$ along a sequence $\varepsilon, \eta \rightarrow 0$ and so

$$
1 \leq F\left(y_{0}, p_{0}\right)=\lim _{\varepsilon, \eta \rightarrow 0} F\left(y, J_{2}\left[\psi_{2}\right](y)\right) \leq a,
$$

which is a contradiction.

To conclude the proof, it remains to establish (29).

Proof of (29): Suppose for some $\eta$ and some sequence $\varepsilon_{m} \rightarrow 0$ that $\frac{1}{\varepsilon_{m}}\left|y_{m}^{*}-y_{m}\right|^{2} \rightarrow d$ where $y_{m}:=y_{\varepsilon_{m}, \eta}$ and $y_{m}^{*}:=y_{\varepsilon_{m}, \eta}^{*}$. (Note that $\frac{1}{\varepsilon}\left|x^{*}-x\right|^{2} \leq C$, so this assumption makes sense.) We need to show that $d \leq \eta$.

Let $\tau_{m}=\tau\left(\varepsilon_{m}, \eta\right)$. Without loss of generality, we assume further that $y_{m} \rightarrow y_{0}$ and $\tau_{m} \rightarrow \tau_{0}$. By the convergence of $y_{m}$ and of $\frac{1}{\varepsilon_{m}}\left|y_{m}^{*}-y_{m}\right|^{2}$, we have that $y_{m}^{*} \rightarrow y_{0}$. Thus, by the upper semi-continuity of $\psi_{1}$, we have

$$
\limsup _{m \rightarrow \infty} \psi_{1}\left(y_{m}^{*}\right) \leq \psi_{1}\left(y_{0}\right)
$$

Hence, by (21), (24) and the left half of (26), we have

$$
\begin{aligned}
0 & \leq \limsup _{m \rightarrow \infty} \frac{1}{\varepsilon_{m}}\left|y_{m}^{*}-y_{m}\right|^{2} \stackrel{\text { (21) }}{=} \limsup _{m \rightarrow \infty}\left(\psi_{1}\left(y_{m}^{*}\right)-\hat{\psi}_{\varepsilon_{m}}\left(y_{m}\right)\right) \\
& \stackrel{(26)}{\leq} \limsup _{m \rightarrow \infty}\left(\psi_{1}\left(y_{m}^{*}\right)-\psi_{2}\left(y_{m}\right)+\tau_{m}\right) \\
& \stackrel{(24)}{\leq} \psi_{1}\left(y_{0}\right)-\psi_{2}\left(y_{0}\right)+\eta=\lim _{m \rightarrow \infty}\left(\hat{\psi}_{\varepsilon_{m}}\left(y_{0}\right)-\psi_{2}\left(y_{0}\right)\right)+\eta \\
& \leq \lim _{m \rightarrow \infty} \sup _{A}\left(\hat{\psi}_{\varepsilon_{m}}-\psi_{2}\right)+\eta \leq \sup _{A}\left(\psi_{1}-\psi_{2}\right)+\eta=\eta .
\end{aligned}
$$

This proves (29) and concludes the proof.

By analogous arguments, we have:

Proposition 2.7. Let $\Omega$ be an open, connected subset of $\mathbb{R}^{n}, n \geq 1$, $\mathscr{U}$ be a nonempty open subset of $\bar{\Omega} \times \mathbb{R} \times \mathbb{R}^{n} \times \mathrm{Sym}_{n}$ and $F \in C^{0}(\overline{\mathscr{U}})$ satisfying (10) and (14). Assume that 
(i) $\psi_{1} \in C_{\mathrm{loc}}^{1,1}(\Omega ; \mathbb{R})$ and $\psi_{2} \in L S C(\Omega \cup\{\infty\})$ satisfy for some constant $a^{\prime}>1$,

$$
F\left(x, J_{2}\left[\psi_{1}\right]\right) \geq a^{\prime} \text { and } F\left(x, J_{2}\left[\psi_{2}\right]\right) \leq 1 \text { in } \Omega \text { in the viscosity sense, }
$$

(ii) $\psi_{1} \leq \psi_{2}$ in $\Omega$ and $\psi_{1}<\psi_{2}$ near $\partial \Omega$.

Then $\psi_{1}<\psi_{2}$ in $\Omega$.

Proof. We argue as in the proof of Proposition 2.6, exchanging the roles of $\psi_{1}$ and $\psi_{2}$ and sup-convolution and inf-convolution.

Assume by contradiction that there exists some $\hat{x} \in \Omega$ such that $\psi_{1}(\hat{x})=\psi_{2}(\hat{x})$.

Step 1: We regularize $\psi_{2}$ by using inf-convolution.

Take some bounded domain $A$ containing $\hat{x}$ such that $\bar{A} \subset \Omega$ and $\psi_{1}<\psi_{2}$ on $\partial A$.

We define, for small $\varepsilon>0$ and $x \in A$,

$$
\hat{\psi}^{\varepsilon}(x)=\inf _{y \in \Omega}\left(\psi_{2}(y)+\frac{1}{\varepsilon}|x-y|^{2}\right) .
$$

It is well-known that $\hat{\psi}^{\varepsilon} \leq \psi_{2}, \hat{\psi}^{\varepsilon}$ is semi-concave, $\nabla^{2} \hat{\psi}^{\varepsilon} \leq \frac{2}{\varepsilon} I$ a.e. in $A$, and $\hat{\psi}^{\varepsilon}$ converges monotonically to $\psi_{2}$ as $\varepsilon \rightarrow 0$. Furthermore, for every $x \in A$, there exists $x_{*}=x_{*}(\varepsilon, x)$ such that

$$
\hat{\psi}^{\varepsilon}(x)=\psi_{2}\left(x_{*}\right)+\frac{1}{\varepsilon}\left|x-x_{*}\right|^{2} .
$$

We note that if $x$ is a point where $\hat{\psi}^{\varepsilon}$ is punctually second order differentiable, then $\psi_{2}$ 'can be touched from below' at $x_{*}$ by a quadratic polynomial:

$$
\psi_{2}\left(x_{*}+z\right) \geq \hat{\psi}^{\varepsilon}(x)-\frac{1}{\varepsilon}\left|x_{*}-x\right|^{2}+\nabla \hat{\psi}^{\varepsilon}(x) \cdot z+\frac{1}{2} z^{T} \nabla^{2} \hat{\psi}^{\varepsilon}(x) z+o\left(|z|^{2}\right), \quad \text { as } z \rightarrow 0,
$$

which is a consequence of the inequalities

$$
\begin{aligned}
& \hat{\psi}^{\varepsilon}(x+z) \geq \hat{\psi}^{\varepsilon}(x)+\nabla \hat{\psi}^{\varepsilon}(x) \cdot z+\frac{1}{2} z^{T} \nabla^{2} \hat{\psi}^{\varepsilon}(x) z+o\left(|z|^{2}\right), \quad \text { as } z \rightarrow 0, \\
& \hat{\psi}^{\varepsilon}(x+z) \leq \psi_{2}\left(x_{*}+z\right)+\frac{1}{\varepsilon}\left|x_{*}-x\right|^{2} .
\end{aligned}
$$

(Here we have used the definition of $\hat{\psi}^{\varepsilon}$ in the last inequality.)

An immediate consequence of (32)-(33) and the fact that $\psi_{2}$ is a super-solution of (9) is that either

$$
\left(x_{*}, \hat{\psi}^{\varepsilon}(x)-\frac{1}{\varepsilon}\left|x_{*}-x\right|^{2}, \nabla \hat{\psi}^{\varepsilon}(x), \nabla^{2} \hat{\psi}^{\varepsilon}(x)\right) \notin \overline{\mathscr{U}}
$$


or

$$
F\left(x_{*}, \hat{\psi}^{\varepsilon}(x)-\frac{1}{\varepsilon}\left|x_{*}-x\right|^{2}, \nabla \hat{\psi}^{\varepsilon}(x), \nabla^{2} \hat{\psi}^{\varepsilon}(x)\right) \leq 1 .
$$

Step 2: We proceed to derive a contradiction as in [33].

For small $\eta>0$, let $\tau=\tau(\varepsilon, \eta)$ be such that

$$
\eta=\sup _{A}\left(\psi_{1}-\hat{\psi}^{\varepsilon}+\tau\right) .
$$

Then

$$
\begin{aligned}
& \tau=\psi_{1}(\hat{x})-\psi_{2}(\hat{x})+\tau \leq \psi_{1}(\hat{x})-\hat{\psi}^{\varepsilon}(\hat{x})+\tau \leq \eta \\
& \tau=\eta-\sup _{A}\left(\psi_{1}-\hat{\psi}^{\varepsilon}\right) \geq \eta-\sup _{A}\left(\psi_{2}-\hat{\psi}^{\varepsilon}\right) .
\end{aligned}
$$

Suppose that $\varepsilon$ and $\eta$ are sufficiently small so that $\xi:=\psi_{1}-\hat{\psi}^{\varepsilon}+\tau$ is negative on $\partial A$. Let $\Gamma_{\xi^{+}}$denote the concave envelop of $\xi^{+}=\max \{\xi, 0\}$. Since $\xi$ is semi-convex and $\xi \leq 0$ on $\partial A$, we have by [8,Lemma 3.5] that

$$
\int_{\left\{\xi=\Gamma_{\xi^{+}}\right\}} \operatorname{det}\left(-\nabla^{2} \Gamma_{\xi^{+}}\right) \geq \frac{1}{C(\Omega)}\left(\sup _{\Omega} \xi\right)^{n}>0 .
$$

In particular, the set $\left\{\xi=\Gamma_{\xi^{+}}\right\}$has positive measure. Recall that $\hat{\psi}^{\varepsilon}$ and $\psi_{1}$ are almost everywhere punctually second order differentiable, we can find $y=y_{\varepsilon, \eta} \in$ $\left\{\xi=\Gamma_{\xi^{+}}\right\}$such that $\hat{\psi}^{\varepsilon}$ and $\psi_{1}$ are punctually second order differentiable at $y$, $\left|J_{2}\left[\psi_{1}\right](y)\right| \leq C\left\|\psi_{1}\right\|_{C^{1,1}(\bar{A})}$,

$$
\begin{gathered}
0<\xi(y)=\psi_{1}(y)-\hat{\psi}^{\varepsilon}(y)+\tau \leq \eta, \\
|\nabla \xi(y)|=\left|\nabla \psi_{1}(y)-\nabla \hat{\psi}^{\varepsilon}(y)\right| \leq C \eta, \\
\nabla^{2} \xi(y)=\nabla^{2} \psi_{1}(y)-\nabla^{2} \hat{\psi}^{\varepsilon}(y) \leq 0,
\end{gathered}
$$

and

$$
\left(y, J_{2}\left[\psi_{1}\right](y)\right) \in \overline{\mathscr{U}}, \quad F\left(y, J_{2}\left[\psi_{1}\right](y)\right) \geq a^{\prime}
$$

We claim that

$$
\liminf _{\varepsilon \rightarrow 0} \frac{1}{\varepsilon}\left|y_{*}-y\right|^{2} \leq \eta
$$

where $y_{*}=x_{*}(\varepsilon, y)$ and $x_{*}$ is defined in (32).

Let us assume (42) for now and go on with the proof. As in Case 1, we may assume that $\left(y, J_{2}\left[\psi_{1}\right](y)\right) \rightarrow\left(y_{0}, p_{0}\right)$ as $\varepsilon, \eta \rightarrow 0$. By (41), $F\left(y_{0}, p_{0}\right) \geq a^{\prime}$ and so by (14), $\left(y_{0}, p_{0}\right) \in \mathscr{U}$. Also, by (36), (37), (38) and (39),

$$
\left(y_{*}, \hat{\psi}^{\varepsilon}(y)-\frac{1}{\varepsilon}\left|y_{*}-y\right|^{2}, \nabla \hat{\psi}^{\varepsilon}(y), \nabla^{2} \psi_{1}(y)\right) \rightarrow\left(y_{0}, p_{0}\right) \text { as } \varepsilon, \eta \rightarrow 0,
$$


and so

$$
\left(y_{*}, \hat{\psi}^{\varepsilon}(y)-\frac{1}{\varepsilon}\left|y_{*}-y\right|^{2}, \nabla \hat{\psi}^{\varepsilon}(y), \nabla^{2} \psi_{1}(y)\right) \in \mathscr{U} \text { along a sequence } \varepsilon, \eta \rightarrow 0,
$$

Now, we have by (10) and (40) that (35) holds at $x=y$ and so

$$
\begin{aligned}
1 & \stackrel{\text { (35) }}{\geq} F\left(y_{*}, \hat{\psi}^{\varepsilon}(y)-\frac{1}{\varepsilon}\left|y_{*}-y\right|^{2}, \nabla \hat{\psi}^{\varepsilon}(y), \nabla^{2} \hat{\psi}^{\varepsilon}(y)\right) \\
& \stackrel{\text { (40) }}{\geq} F\left(y_{*}, \hat{\psi}^{\varepsilon}(y)-\frac{1}{\varepsilon}\left|y_{*}-y\right|^{2}, \nabla \hat{\psi}^{\varepsilon}(y), \nabla^{2} \psi_{1}(y)\right) \\
& =F\left(y, J_{2}\left[\psi_{1}\right](y)\right)+o_{\varepsilon, \eta}(1) \\
& \stackrel{\text { (41) }}{\geq} a^{\prime}+o_{\varepsilon, \eta}(1),
\end{aligned}
$$

where $\lim _{\varepsilon, \eta \rightarrow 0} o_{\varepsilon, \eta}(1)=0$ and where we have used the (local uniform) continuity of $F$ in the second-to-last equality. This gives a contradiction as $a^{\prime}>1$.

To conclude the proof, it remains to establish (42).

Proof of (42): Suppose for some $\eta>0$ and some sequence $\varepsilon_{m} \rightarrow 0$ that $\frac{1}{\varepsilon_{m}} \mid\left(y_{m}\right)_{*}$

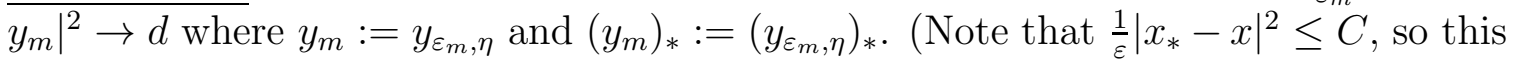
assumption makes sense.) We need to show that $d \leq \eta$.

Let $\tau_{m}=\tau\left(\varepsilon_{m}, \eta\right)$. Without loss of generality, we assume further that $y_{m} \rightarrow y_{0}$ and $\tau_{m} \rightarrow \tau_{0}$. By the convergence of $y_{m}$ and of $\frac{1}{\varepsilon_{m}}\left|\left(y_{m}\right)_{*}-y_{m}\right|^{2}$, we have that $\left(y_{m}\right)_{*} \rightarrow y_{0}$. Thus, by the lower semi-continuity of $\psi_{2}$, we have

$$
\liminf _{m \rightarrow \infty} \psi_{2}\left(\left(y_{m}\right)_{*}\right) \geq \psi_{2}\left(y_{0}\right) .
$$

Hence, by (32), (36) and the left half of (38), we have

$$
\begin{aligned}
0 & \leq \liminf _{m \rightarrow \infty} \frac{1}{\varepsilon_{m}}\left|\left(y_{m}\right)_{*}-y_{m}\right|^{2} \stackrel{\sqrt[32]{=}}{=} \liminf _{m \rightarrow \infty}\left(\hat{\psi}^{\varepsilon_{m}}\left(y_{m}\right)-\psi_{2}\left(\left(y_{m}\right)_{*}\right)\right) \\
& \stackrel{38}{\leq} \liminf _{m \rightarrow \infty}\left(\psi_{1}\left(y_{m}\right)-\psi_{2}\left(\left(y_{m}\right)_{*}\right)+\tau_{m}\right) \\
& \stackrel{36}{\leq} \psi_{1}\left(y_{0}\right)-\psi_{2}\left(y_{0}\right)+\eta=\lim _{m \rightarrow \infty}\left(\psi_{1}\left(y_{0}\right)-\hat{\psi}^{\varepsilon_{m}}\left(y_{0}\right)\right)+\eta \\
& \leq \lim _{m \rightarrow \infty} \sup _{A}\left(\psi_{1}-\hat{\psi}^{\varepsilon_{m}}\right)+\eta \leq \sup _{A}\left(\psi_{1}-\psi_{2}\right)+\eta=\eta .
\end{aligned}
$$

This proves (42) and concludes the proof.

We now give the 
Proof of Theorem 2.3. Arguing by contradiction, suppose the conclusion is wrong, then we can find a closed ball $\bar{B} \subset \Omega$ of radius $R>0$ and a point $\hat{x} \in \partial B$ such that

$$
\psi_{1}<\psi_{2} \text { in } \bar{B} \backslash\{\hat{x}\} \text { and } \psi_{1}(\hat{x})=\psi_{2}(\hat{x}) .
$$

Without loss of generality, we assume the center of $B$ is the origin.

Case 1: Consider first the case $\psi_{2}$ is $C^{1,1}$.

In the proof, $C$ denotes some generic constant which may vary from lines to lines but depends only on an upper bound for $\left\|\psi_{2}\right\|_{C^{1,1}(\bar{\Omega})}, \Omega$ and $(F, \mathscr{U})$.

In view of Proposition 2.6, it suffices to deform $\psi_{2}$ to a strict super-solution $\tilde{\psi}_{2}$ in some open ball $A$ around $\hat{x}$ such that $\tilde{\psi}_{2}>\psi_{1}$ on $\partial A$ and $\inf _{A}\left(\tilde{\psi}_{2}-\psi_{1}\right)=0$. We adapt the argument in [9, which assumes that $\psi_{2}$ is $C^{2}$.

Using that $\psi_{2}$ is $C^{1,1}$, a theorem of Alexandrov, Buselman and Feller (see e.g. 8, Theorem 1.5]) and Lemma 2.5. we can find some $\Lambda>0$ and a set $Z$ of zero measure such that $\psi_{2}$ is punctually second order differentiable in $\Omega \backslash Z$,

$$
\left|J_{2}\left[\psi_{2}\right]\right| \leq \Lambda \text { in } \Omega \backslash Z \text {. }
$$

and

$$
\text { either }\left(x, J_{2}\left[\psi_{2}\right](x)\right) \notin \overline{\mathscr{U}} \text { or } F\left(x, J_{2}\left[\psi_{2}\right](x)\right) \leq 1 \text { in } \Omega \backslash Z \text {. }
$$

By (11), there is some small constant $\theta_{0}>0$

$$
F(x, s, p, M) \leq 1-2 \theta_{0} \text { for all } x \in \bar{\Omega},(s, p, M) \in \partial \mathscr{U}_{x},|s|+|p|+|M| \leq \Lambda+2 .
$$

Hence

$$
\mathscr{K}:=\left\{(x, s, p, M) \in \mathscr{U}: F(x, s, p, M) \geq 1-\theta_{0}, x \in \bar{\Omega},|s|+|p|+|M| \leq \Lambda+1\right\}
$$

and

$$
\begin{aligned}
\mathscr{K}^{\prime}:=\left\{(x, s, p, M) \in \mathscr{U}: F(x, s, p, M) \geq 1-\theta_{0} / 2, x \in \bar{\Omega},\right. \\
|s|+|p|+|M| \leq \Lambda+1 / 2\} \subset \mathscr{K}
\end{aligned}
$$

are compact.

For $\alpha>1, \mu>0$ and $\tau>0$ which will be fixed later, let

$$
\begin{aligned}
E(x) & =E_{\alpha}(x)=e^{-\alpha|x|^{2}}, \\
h(x) & =h_{\alpha}(x)=e^{-\alpha|x|^{2}}-e^{-\alpha R^{2}}, \\
\zeta(x) & =\zeta_{\alpha}(x)=\cos \left(\alpha^{1 / 2}\left(x_{1}-\hat{x}_{1}\right)\right), \\
\tilde{\psi}_{\mu, \tau} & =\psi_{2}-\mu(h-\tau) \zeta .
\end{aligned}
$$


Let $A$ be a ball centered at $\hat{x}$ such that $\zeta>\frac{1}{2}$ in $A$ and $\tau_{0}=\sup _{A} h>0$.

It is clear that, for $0 \leq \tau \leq \tau_{0}$ and all sufficiently small $\mu$,

$$
\tilde{\psi}_{\mu, \tau}>\psi_{1} \text { on } \partial A \text {. }
$$

We compute

$$
\begin{aligned}
\nabla \tilde{\psi}_{\mu, \tau}(x)= & \nabla \psi_{2}(x)+2 \mu \alpha E \zeta x+\mu \alpha^{1 / 2}(h-\tau) \sin \left(\alpha^{1 / 2}\left(x_{1}-\hat{x}_{1}\right)\right) e_{1}, \\
\nabla^{2} \tilde{\psi}_{\mu, \tau}(x)=\nabla^{2} \psi_{2}(x)-2 \mu \alpha E \zeta(2 \alpha x \otimes x-I) & \\
& -2 \mu \alpha^{3 / 2} E \sin \left(\alpha^{1 / 2}\left(x_{1}-\hat{x}_{1}\right)\left(e_{1} \otimes x+x \otimes e_{1}\right)\right. \\
& +\mu \alpha(h-\tau) \zeta e_{1} \otimes e_{1} .
\end{aligned}
$$

We thus have

$$
J_{2}\left[\tilde{\psi}_{\mu, \tau}\right](x)=J_{2}\left[\psi_{2}\right](x)-\left(0,0,4 \mu \alpha^{2} E \zeta x \otimes x+\mu \tau \alpha \zeta e_{1} \otimes e_{1}\right)+O\left(\mu\left(\alpha^{3 / 2} E+\alpha^{1 / 2} \tau\right)\right) .
$$

Now if $x \in A \backslash Z$ is such that

$$
\begin{aligned}
\left(x, J_{2}\left[\tilde{\psi}_{\mu, \tau}\right](x)\right),\left(x, J_{2}\left[\psi_{2}\right](x)\right) & \\
& \text { and }\left(x, J_{2}\left[\tilde{\psi}_{\mu, \tau}\right]+\left(0,0,4 \mu \alpha^{2} E \zeta x \otimes x+\mu \tau \alpha \zeta e_{1} \otimes e_{1}\right) \text { lie in } \mathscr{K},\right.
\end{aligned}
$$

then

$$
\begin{aligned}
F(x, & \left.J_{2}\left[\psi_{2}\right]\right)+C \mu \alpha^{3 / 2} E+C \mu \tau \alpha^{1 / 2} \\
& \quad \stackrel{[133)}{\geq} F\left(x, J_{2}\left[\tilde{\psi}_{\mu, \tau}\right]+\left(0,0,4 \mu \alpha^{2} E \zeta x \otimes x+\mu \tau \alpha \zeta e_{1} \otimes e_{1}\right)\right) \\
& \stackrel{\text { (12) }}{\geq} F\left(x, J_{2}\left[\tilde{\psi}_{\mu, \tau}\right]\right)+\frac{1}{C} \mu \alpha^{2} E+\frac{1}{C} \mu \tau \alpha,
\end{aligned}
$$

and so, by selecting a sufficiently large $\alpha$, we thus obtain for some $\beta>0$ and all sufficiently small $\mu$,

$$
F\left(x, J_{2}\left[\tilde{\psi}_{\mu, \tau}\right]\right) \leq F\left(x, J_{2}\left[\psi_{2}\right]\right)-\beta \mu \stackrel{(444)}{\leq} 1-\beta \mu .
$$

Now for every $x \in A \backslash Z$ satisfying $J_{2}\left[\tilde{\psi}_{\mu, \tau}\right](x) \in \mathscr{U}_{x}$ and $F\left(x, J_{2}\left[\tilde{\psi}_{\mu, \tau}\right](x)\right) \geq$ $1-\theta_{0} / 2$, we have, in view of (43), that $\left|J_{2}\left[\tilde{\psi}_{\mu, \tau}\right](x)\right| \leq \Lambda+1 / 2$ and so $\left(x, J_{2}\left[\tilde{\psi}_{\mu, \tau}\right](x)\right)$ lies in $\mathscr{K}^{\prime}$ for all small $\mu$. By squeezing $\mu$ further, we then have that $\left(x, J_{2}\left[\psi_{2}\right](x)\right)$ and $\left(x, J_{2}\left[\tilde{\psi}_{\mu, \tau}\right]+\left(0,0,4 \mu \alpha^{2} E \zeta x \otimes x+\mu \tau \alpha \zeta e_{1} \otimes e_{1}\right)\right.$ lie in $\mathscr{K}$. In particular, (466) holds.

Taking $\tilde{\beta}=\min \left(\beta, \frac{\theta_{0}}{2 \mu}\right)$, we thus obtain that either $J_{2}\left[\tilde{\psi}_{\mu, \tau}\right](x) \notin \overline{\mathscr{U}}_{x}$ or $F\left(x, J_{2}\left[\tilde{\psi}_{\mu, \tau}\right](x)\right) \leq 1-\tilde{\beta} \mu$ in $A \backslash Z$. 
Noting that

$$
\inf _{A}\left(\tilde{\psi}_{\mu, 0}-\psi_{1}\right) \leq 0 \leq \inf _{A}\left(\tilde{\psi}_{\mu, \tau_{0}}-\psi_{1}\right)
$$

we can select $\tau_{1} \in\left[0, \tau_{0}\right]$ such that

$$
\inf _{A}\left(\tilde{\psi}_{\mu, \tau_{1}}-\psi_{1}\right)=0
$$

The desired $\tilde{\psi}_{2}$ is taken to be $\tilde{\psi}_{\mu, \tau_{1}}$. The conclusion follows from Proposition 2.6.

Case 2: Consider now the case $\psi_{1}$ is $C^{1,1}$.

The proof is similar. $C$ will now denote some generic constant which depends only on an upper bound for $\left\|\psi_{1}\right\|_{C^{1,1}(\bar{\Omega})}, \Omega$ and $(F, \mathscr{U})$.

In view of Proposition 2.7, it suffices to deform $\psi_{1}$ to a strict sub-solution $\tilde{\psi}_{1}$ in some open ball $A$ around $\hat{x}$ such that $\psi_{2}>\tilde{\psi}_{1}$ on $\partial A$ and $\inf _{A}\left(\psi_{2}-\tilde{\psi}_{1}\right)=0$.

Using that $\psi_{1}$ is $C^{1,1}$, a theorem of Alexandrov, Buselman and Feller and Lemma 2.5. we can find some $\Lambda>0$ and a set $Z$ of zero measure such that $\psi_{1}$ is puntually second order differentiable in $\Omega \backslash Z$,

$$
\left|J_{2}\left[\psi_{1}\right]\right| \leq \Lambda \text { in } \Omega \backslash Z
$$

and, by (11),

$$
\left(x, J_{2}\left[\psi_{1}\right](x)\right) \in \mathscr{U} \text { and } F\left(x, J_{2}\left[\psi_{1}\right](x)\right) \geq 1 \text { in } \Omega \backslash Z .
$$

For $\alpha>1, \mu>0$ and $\tau>0$ which will be fixed later, let $E, h, \zeta, A, \tau_{0}$ be as in Case 1 , and amend the definition of $\tilde{\psi}_{\mu, \tau}$ to

$$
\tilde{\psi}_{\mu, \tau}=\psi_{1}+\mu(h-\tau) \zeta
$$

It is clear that, for $0 \leq \tau \leq \tau_{0}$ and all sufficiently small $\mu$,

$$
\tilde{\psi}_{\mu, \tau}<\psi_{2} \text { on } \partial A \text {. }
$$

As before, we have

$$
J_{2}\left[\tilde{\psi}_{\mu, \tau}\right](x)=J_{2}\left[\psi_{1}\right](x)+\left(0,0,4 \mu \alpha^{2} E \zeta x \otimes x+\mu \tau \alpha \zeta e_{1} \otimes e_{1}\right)+O\left(\mu\left(\alpha^{3 / 2} E+\alpha^{1 / 2} \tau\right)\right) .
$$

It is clear from (47) that $\left(x, J_{2}\left[\psi_{1}\right](x)\right)$ belongs to $\mathscr{K}^{\prime}$ for all $x \in A \backslash Z$. We thus have for all sufficiently small $\mu$ and $x \in A \backslash Z$ that

$$
\begin{aligned}
&\left(x, J_{2}\left[\tilde{\psi}_{\mu, \tau}\right]\right), \quad\left(x, J_{2}\left[\psi_{1}\right]\right) \\
& \text { and }\left(x, J_{2}\left[\tilde{\psi}_{\mu, \tau}\right]-\left(0,0,4 \mu \alpha^{2} E \zeta x \otimes x+\mu \tau \alpha \zeta e_{1} \otimes e_{1}\right)\right) \text { lie in } \mathscr{K} .
\end{aligned}
$$


Therefore,

$$
\begin{aligned}
& F\left(x, J_{2}\left[\psi_{1}\right]\right)-C \mu \alpha^{3 / 2} E-C \mu \tau \alpha^{1 / 2} \\
& \stackrel{\text { (13) }}{\leq} F\left(x, J_{2}\left[\tilde{\psi}_{\mu, \tau}\right]-\left(0,0,4 \mu \alpha^{2} E \zeta x \otimes x+\mu \tau \alpha \zeta e_{1} \otimes e_{1}\right)\right) \\
& \stackrel{(12)}{\leq} F\left(x, J_{2}\left[\tilde{\psi}_{\mu, \tau}\right]\right)-\frac{1}{C} \mu \alpha^{2} E-\frac{1}{C} \mu \tau \alpha,
\end{aligned}
$$

and so, by selecting a sufficiently large $\alpha$, we thus obtain for some $\beta>0$ and all sufficiently small $\mu$,

$$
F\left(x, J_{2}\left[\tilde{\psi}_{\mu, \tau}\right]\right) \geq F\left(x, J_{2}\left[\psi_{1}\right]\right)+\beta \mu \stackrel{\text { (47]) }}{\geq} 1+\beta \mu .
$$

Noting that

$$
\inf _{A}\left(\psi_{2}-\tilde{\psi}_{\mu, 0}\right) \leq 0 \leq \inf _{A}\left(\psi_{2}-\tilde{\psi}_{\mu, \tau_{0}}\right),
$$

we can select $\tau_{1} \in\left[0, \tau_{0}\right]$ such that

$$
\inf _{A}\left(\psi_{2}-\tilde{\psi}_{\mu, \tau_{1}}\right)=0
$$

The desired $\tilde{\psi}_{1}$ is taken to be $\tilde{\psi}_{\mu, \tau_{1}}$. The conclusion follows from Proposition 2.7 (and Lemma 2.5).

\subsection{Proof of the Hopf Lemma}

Proof of Theorem 2.4. We will only consider the case that $\psi_{2}$ is $C^{1,1}$, since the case when $\psi_{1}$ is $C^{1,1}$ can be treated similarly.

Since $\partial \Omega$ is $C^{2}$ near $\hat{x}$, we can find a ball $B$ such that $\bar{B} \subset \Omega \cup\{\hat{x}\}$ and $\hat{x} \in \partial B$. Thus we may assume without loss of generality that $\Omega=B$ is a ball centered at the origin, $u_{1}$ and $u_{2}$ are defined on $\bar{B}$ and $u_{1}<u_{2}$ in $\bar{B} \backslash\{\hat{x}\}$.

The function $\tilde{\psi}_{\mu, \tau}=\psi_{2}-\mu(h-\tau) \zeta$ defined by (45) in the proof of Theorem 2.3 satisfies for some open ball $A$ centered at $\hat{x}$, some constant $\beta>0$, and all $0 \leq \tau \leq$ $\tau_{0}:=\sup _{A \cap B} h$ that

$$
\text { either } J_{2}\left[\tilde{\psi}_{\mu, \tau}\right](x) \notin \overline{\mathscr{U}}_{x} \text { or } F\left(x, J_{2}\left[\tilde{\psi}_{\mu, \tau}\right](x)\right) \leq 1-\beta \mu \text { a.e. in } A \cap B \text {. }
$$

If $\tilde{\psi}_{\mu, 0} \geq \psi_{1}$ in $A \cap B$ for some $\mu>0$, we are done by the explicit form of $h$. Suppose otherwise that

$$
\inf _{A \cap B}\left(\tilde{\psi}_{\mu, 0}-\psi_{1}\right)<0
$$

Noting that

$$
0 \leq \inf _{A \cap B}\left(\tilde{\psi}_{\mu, \tau_{0}}-\psi_{1}\right)
$$


we can find $\tau_{1} \in\left(0, \tau_{0}\right]$ such that

$$
\inf _{A \cap B}\left(\tilde{\psi}_{\mu, \tau_{1}}-\psi_{1}\right)=0 .
$$

Recall the definition of $h$, we have also that

$$
\inf _{\partial(A \cap B)}\left(\tilde{\psi}_{\mu, \tau_{1}}-\psi_{1}\right)>0 .
$$

Recalling (49), we obtain a contradiction to Proposition 2.6,

\section{Proof of the Liouville theorem}

In this section, we prove our Liouville theorem. Let us start with some preliminary. Define

$$
U=\left\{M \in \operatorname{Sym}_{n}: \lambda(U) \in \Gamma\right\}
$$

and

$$
F(M)=f(\lambda(M))
$$

By (21)-(17), we have

(i) $(F, U)$ is elliptic, i.e.

$$
\text { if } M \in U \text { and } N \geq 0 \text {, then } M+N \in U \text { and } F(M+N) \geq F(M) .
$$

(ii) $(F, U)$ is locally strictly elliptic, i.e. for any compact subset $K$ of $U$, there is some constant $\delta(K)>0$ such that

$$
F(M+N)-F(M) \geq \delta(K)|N| \text { for all } M \in K, N \geq 0 .
$$

(iii) $F$ is locally Lipschitz, i.e. for any compact subset $K$ of $U$, there is some constant $C(K)>0$ such that

$$
\left|F\left(M^{\prime}\right)-F(M)\right| \leq C(K)\left|M^{\prime}-M\right| \text { for all } M, M^{\prime} \in K .
$$

(iv) The 1-superlevel set of $F$ stays in $U$, namely

$$
F^{-1}([1, \infty)) \subset U \text {. }
$$

(v) $(F, U)$ is invariant under the orthogonal group $O(n)$, i.e.

$$
\text { if } M \in U \text { and } R \in O(n) \text {, then } R^{t} M R \in U \text { and } F\left(R^{t} M R\right)=F(M) \text {. }
$$


(vi) $U$ satisfies

$$
\operatorname{tr}(M) \geq 0 \text { for all } M \in U \text {. }
$$

From (50)-(53), we see that the strong comparison principle (Theorem 2.3) and the Hopf Lemma (Theorem 2.4) are applicable to the equation $F\left(A^{u}\right)=1$ by setting $\psi=-\ln u$.

An essential ingredient for our proof is a conformal property of the conformal Hessian $A^{w}$, inherited from the conformal structure of $\mathbb{R}^{n}$. Recall that a map $\varphi: \mathbb{R}^{n} \cup$ $\{\infty\} \rightarrow \mathbb{R}^{n} \cup\{\infty\}$ is called a Möbius transformation if it is the composition of finitely many translations, dilations and inversions. Now if $\varphi$ is a Möbius transformation and if we set $w_{\varphi}=\left|J_{\varphi}\right|^{\frac{n-2}{2 n}} w \circ \varphi$ where $J_{\varphi}$ is the Jacobian of $\varphi$, then

$$
A^{w_{\varphi}}(x)=O_{\varphi}(x)^{t} A^{w}(\varphi(x)) O_{\varphi}(x)
$$

for some orthogonal $n \times n$ matrix $O_{\varphi}(x)$. In particular, by (54),

$$
F\left(A^{w_{\varphi}}(x)\right)=F\left(A^{w}(\varphi(x))\right) .
$$

Proof of Theorem 1.3. Having established the Hopf Lemma and the strong comparison principle, we can follow the proof of [32, Theorem 1.1], which draws on ideas from [27], to reach the conclusion. We give a sketch here for readers' convenience. For details, see [32, Section 2].

We use the method of moving spheres. For a function $w$ defined on a subset of $\mathbb{R}^{n}$, we define

$$
w_{x, \lambda}(y)=\frac{\lambda^{n-2}}{|y-x|^{n-2}} w\left(x+\frac{\lambda^{2}(y-x)}{|y-x|^{2}}\right)
$$

wherever the expression makes sense.

Step 1: We set up the moving sphere method.

Since $v_{k}$ is locally uniformly bounded, local gradient estimates (see e.g. [32, Theorem 2.1], [30, Theorem 1.10]), imply that $\left|\nabla v_{k}\right|$ is locally uniformly bounded and so $v_{k}$ converges to $v$ in $C_{l o c}^{0, \alpha}\left(\mathbb{R}^{n}\right)$ and $v \in C_{l o c}^{0,1}\left(\mathbb{R}^{n}\right)$.

We note that, by (55), $v$ is super-harmonic. Thus, by the positivity of $v$ and the maximum principle, we have

$$
v(y) \geq \frac{1}{C}(1+|y|)^{2-n} \text { for all } y \in \mathbb{R}^{n},
$$

and so we may also assume without loss of generality that

$$
\left\|v_{k}-v\right\|_{C^{0}\left(B_{R_{k}}(0)\right)} \leq R_{k}^{-n} \text { and } v_{k}(y) \geq \frac{1}{C}(1+|y|)^{2-n} \text { for all } y \in B_{R_{k}}(0) .
$$


Using (58) and the local uniform boundedness of $\left|\nabla v_{k}\right|$, one can show that there is a function $\lambda^{(0)}: \mathbb{R}^{n} \rightarrow(0, \infty)$ such that for all $k$,

$$
\left(v_{k}\right)_{x, \lambda} \leq v_{k} \text { in } B_{R_{k}}(0) \backslash B_{\lambda}(x), \forall 0<\lambda<\lambda^{(0)}(x),|x|<R_{k} / 5 .
$$

See [32, Lemma 2.2].

Define, for $|x|<R_{k} / 5$,

$$
\bar{\lambda}_{k}(x)=\sup \left\{0<\mu<R_{k} / 5: u_{x, \lambda} \leq u \text { in } B_{R_{k}}(0) \backslash B_{\lambda}(x), \forall 0<\lambda<\mu\right\} .
$$

By (다), $\bar{\lambda}_{k}(x) \in\left[\lambda^{(0)}(x), R_{k} / 5\right]$. Set

$$
\bar{\lambda}(x)=\liminf _{k \rightarrow \infty} \bar{\lambda}_{k}(x) \in\left[\lambda^{(0)}(x), \infty\right] .
$$

$\bar{\lambda}(x)$ is sometimes referred to as the moving sphere radius of $v$ at $x$,

Step 2: We show that if $\bar{\lambda}(x)<\infty$ for some $x \in \mathbb{R}^{n}$, then

$$
\alpha:=\liminf _{|y| \rightarrow \infty}|y|^{n-2} u(y)=\lim _{|y| \rightarrow \infty}|y|^{n-2} v_{x, \bar{\lambda}(x)}(y)=\bar{\lambda}(x)^{n-2} v(x)<\infty .
$$

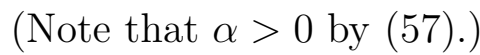

We have

$$
\left(v_{k}\right)_{x, \bar{\lambda}_{k}(x)} \leq v_{k} \text { in } \mathbb{R}^{n} \backslash B_{\bar{\lambda}_{k}(x)}(x),
$$

By the conformal invariance of the conformal Hessian (56) $),\left(v_{k}\right)_{x, \bar{\lambda}_{k}(x)}$ satisfies

$$
F\left(A^{\left.\left(v_{k}\right)_{x, \bar{\lambda}_{k}(x)}\right)}=1 \quad \text { in } \quad \mathbb{R}^{n} \backslash \overline{B_{\bar{\lambda}_{k}(x)}(x)} .\right.
$$

We can now apply the strong comparison principle (Theorem 2.3) and the Hopf Lemma (Theorem 2.4 $)$ to conclude that there exists $y_{k} \in \partial B_{R_{k}}(0)$ such that $\left(v_{k}\right)_{x, \bar{\lambda}_{k}(x)}=$ $v_{k}\left(y_{k}\right)$. (See the proof of [27, Lemma 4.5].)

It follows that

$$
\begin{aligned}
\alpha & \leq \liminf _{k \rightarrow \infty}\left|y_{k}\right|^{n-2} v\left(y_{k}\right)=\liminf _{k \rightarrow \infty}\left|y_{k}\right|^{n-2} v_{k}\left(y_{k}\right) \\
& =\liminf _{k \rightarrow \infty}\left|y_{k}\right|^{n-2}\left(v_{k}\right)_{x, \bar{\lambda}_{k}(x)}\left(y_{k}\right)=(\bar{\lambda}(x))^{n-2} v(x)<\infty .
\end{aligned}
$$

The opposite inequality that $\alpha \geq(\bar{\lambda}(x))^{n-2} v(x)$ is an easy consequence of the inequality $v_{x, \bar{\lambda}(x)} \leq v$ in $\mathbb{R}^{n} \backslash B_{\bar{\lambda}(x)}(x)$. This proves (60).

Step 3: We show that either $v$ is constant or $\bar{\lambda}(x)<\infty$ for all $x \in \mathbb{R}^{n}$.

Suppose that $\bar{\lambda}\left(x_{0}\right)=\infty$ for some $x_{0}$. Then we hve

$$
v_{x_{0}, \lambda} \leq v \text { in } \mathbb{R}^{n} \backslash B_{\lambda}\left(x_{0}\right) \text { for all } \lambda>0 .
$$


It follows that, for every unit vector $e$, the function $r \mapsto r^{\frac{n-2}{2}} v\left(x_{0}+r e\right)$ is nondecreasing. It follows that

$$
r^{n-2} \inf _{\partial B_{r}\left(x_{0}\right)} v \geq r^{\frac{n-2}{2}} \inf _{\partial B_{1}\left(x_{0}\right)} v
$$

and so

$$
\alpha=\liminf _{|y| \rightarrow \infty}|y|^{n-2} v(y)=\infty .
$$

Thus, by Step 2 above, we have $\bar{\lambda}(x)=\infty$ for all $x \in \mathbb{R}^{n}$. This implies that $v$ is constant; see e.g. [28], [32, Lemma C.1]. This implies that $0 \in \Gamma$ and $f(0)=1$.

Step 4: By Steps 2 and 3, it remains to consider the case where, for every $x \in \mathbb{R}^{n}$, there exists $0<\bar{\lambda}(x)<\infty$ such that

(i) $v_{x, \bar{\lambda}(x)} \leq v$ in $\mathbb{R}^{n} \backslash B_{\bar{\lambda}(x)}(x)$,

(ii) and

$$
\alpha=\lim _{|y| \rightarrow \infty}|y|^{n-2} v(y)=\lim _{|y| \rightarrow \infty}|y|^{n-2} v_{x, \bar{\lambda}(x)}(y) .
$$

In some sense, we have a strong comparison principle situation where touching occurs at infinity. If $v$ was $C^{1,1}$, this would imply that $v_{x, \bar{\lambda}(x)} \equiv v$ and so a calculus argument would then show that $v$ has the desired form (see [37, Lemma 11.1]).

Since we have not established the strong comparison principle in $C^{0,1}$ regularity, we resort to a different argument, which was first observed in [27] for $C^{2}$ solution and [29] for $C^{0,1}$ solutions. It turns out that, (i) and (ii) together with the superharmonicity of $v$ imply directly that there exist $a, b>0$ and $x_{0} \in \mathbb{R}^{n}$ such that

$$
u(x)=\left(\frac{a}{1+b^{2}\left|x-x_{0}\right|^{2}}\right)^{\frac{n-2}{2}} .
$$

See [29, 32]. This concludes the proof.

\section{References}

[1] M. E. Amendola, G. Galise, And A. Vitolo, Riesz capacity, maximum principle, and removable sets of fully nonlinear second-order elliptic operators, Differential Integral Equations, 26 (2013), pp. 845-866.

[2] M. BARDI AND F. DA LiO, On the strong maximum principle for fully nonlinear degenerate elliptic equations, Arch. Math. (Basel), 73 (1999), pp. 276-285. 
[3] H. Berestycki, I. Capuzzo Dolcetta, A. Porretta, and L. Rossi, Maximum principle and generalized principal eigenvalue for degenerate elliptic operators, J. Math. Pures Appl. (9), 103 (2015), pp. 1276-1293.

[4] I. Birindelli And F. Demengel, Comparison principle and Liouville type results for singular fully nonlinear operators, Ann. Fac. Sci. Toulouse Math. (6), 13 (2004), pp. 261-287.

[5] _ Eigenvalue, maximum principle and regularity for fully non linear homogeneous operators, Commun. Pure Appl. Anal., 6 (2007), pp. 335-366.

[6] I. Birindelli, G. Galise, and H. Ishit, A family of degenerate elliptic operators: maximum principle and its consequences, Ann. Inst. H. Poincaré Anal. Non Linéaire, 35 (2018), pp. 417-441.

[7] _ - Towards a reversed Faber-Krahn inequality for the truncated Laplacian, preprint, (2018).

[8] L. Caffarelli and X. Cabré, Fully nonlinear elliptic equations, vol. 43 of American Mathematical Society Colloquium Publications, American Mathematical Society, Providence, RI, 1995.

[9] L. Caffarelli, Y. Y. Li, and L. Nirenberg, Some remarks on singular solutions of nonlinear elliptic equations III: viscosity solutions including parabolic operators, Comm. Pure Appl. Math., 66 (2013), pp. 109-143.

[10] L. A. Caffarelli, B. Gidas, And J. Spruck, Asymptotic symmetry and local behavior of semilinear elliptic equations with critical Sobolev growth, Comm. Pure Appl. Math., 42 (1989), pp. 271-297.

[11] S.-Y. A. Chang, M. J. Gursky, And P. C. YAng, Entire solutions of a fully nonlinear equation, in Lectures on partial differential equations, vol. 2 of New Stud. Adv. Math., Int. Press, Somerville, MA, 2003, pp. 43-60.

[12] M. G. Crandall, H. Ishit, And P.-L. Lions, User's guide to viscosity solutions of second order partial differential equations, Bull. Amer. Math. Soc. (N.S.), 27 (1992), pp. 1-67.

[13] I. C. Dolcetta And A. Vitolo, On the maximum principle for viscosity solutions of fully nonlinear elliptic equations in general domains, Matematiche (Catania), 62 (2007), pp. 69-91.

[14] _ - The weak maximum principle for degenerate elliptic operators in unbounded domains, Int. Math. Res. Not. IMRN, (2018), pp. 412-431. 
[15] B. Gidas, W. M. Ni, AND L. NirenberG, Symmetry of positive solutions of nonlinear elliptic equations in $\mathbf{R}^{n}$, in Mathematical analysis and applications, Part A, vol. 7 of Adv. in Math. Suppl. Stud., Academic Press, New York-London, 1981, pp. 369-402.

[16] P. Hartman and L. NirenberG, On spherical image maps whose Jacobians do not change sign, Amer. J. Math., 81 (1959), pp. 901-920.

[17] F. R. Harvey and H. B. LaWson, JR., Existence, uniqueness and removable singularities for nonlinear partial differential equations in geometry, in Surveys in differential geometry. Geometry and topology, vol. 18 of Surv. Differ. Geom., Int. Press, Somerville, MA, 2013, pp. 103-156.

[18] H. ISHII, On uniqueness and existence of viscosity solutions of fully nonlinear second-order elliptic PDEs, Comm. Pure Appl. Math., 42 (1989), pp. 15-45.

[19] H. Ishil AND P.-L. Lions, Viscosity solutions of fully nonlinear second-order elliptic partial differential equations, J. Differential Equations, 83 (1990), pp. 2678.

[20] R. JENSEN, The maximum principle for viscosity solutions of fully nonlinear second order partial differential equations, Arch. Rational Mech. Anal., 101 (1988), pp. 1-27.

[21] B. KaWohl And N. Kutev, Strong maximum principle for semicontinuous viscosity solutions of nonlinear partial differential equations, Arch. Math. (Basel), 70 (1998), pp. 470-478.

[22] — Comparison principle and Lipschitz regularity for viscosity solutions of some classes of nonlinear partial differential equations, Funkcial. Ekvac., 43 (2000), pp. 241-253.

[23] _ Comparison principle for viscosity solutions of fully nonlinear, degenerate elliptic equations, Comm. Partial Differential Equations, 32 (2007), pp. 12091224.

[24] S. Kolke And T. Kosugi, Remarks on the comparison principle for quasilinear PDE with no zeroth order terms, Commun. Pure Appl. Anal., 14 (2015), pp. 133142.

[25] S. KoIKE AND O. LEY, Comparison principle for unbounded viscosity solutions of degenerate elliptic PDEs with gradient superlinear terms, J. Math. Anal. Appl., 381 (2011), pp. 110-120. 
[26] A. Li AND Y. Y. LI, On some conformally invariant fully nonlinear equations, Comm. Pure Appl. Math., 56 (2003), pp. 1416-1464.

[27] — - On some conformally invariant fully nonlinear equations. II. Liouville, Harnack and Yamabe, Acta Math., 195 (2005), pp. 117-154.

[28] Y. Y. LI, Conformally invariant fully nonlinear elliptic equations and isolated singularities, J. Funct. Anal., 233 (2006), pp. 380-425.

[29] — Degenerate conformally invariant fully nonlinear elliptic equations, Arch. Ration. Mech. Anal., 186 (2007), pp. 25-51.

[30] _ Local gradient estimates of solutions to some conformally invariant fully nonlinear equations, Comm. Pure Appl. Math., 62 (2009), pp. 1293-1326. (C. R. Math. Acad. Sci. Paris 343 (2006), no. 4, 249-252).

[31] Y. Y. Li And D. D. Monticelli, On fully nonlinear CR invariant equations on the Heisenberg group, J. Differential Equations, 252 (2012), pp. 1309-1349.

[32] Y. Y. Li And L. NGuYEn, Symmetry, quantitative Liouville theorems and analysis of large solutions of conformally invariant fully nonlinear elliptic equations, Calc. Var. Partial Differential Equations, 56 (2017), pp. Art. 99, 35.

[33] Y. Y. Li, L. NGuyen, And B. Wang, Comparison principles and Lipschitz regularity for some nonlinear degenerate elliptic equations, Calc. Var. Partial Differential Equations, 57 (2018), pp. Art. 96, 29.

[34] Y. Y. Li And L. Nirenberg, A miscellany, in Percorsi incrociati (in ricordo di Vittorio Cafagna), Collana Scientifica di Ateneo, Universita di Salerno, 2010, pp. 193-208. http://arxiv.org/abs/0910.0323.

[35] Y. Y. Li AND B. WANG, Strong comparison principles for some nonlinear degenerate elliptic equations, Acta Math. Sci. Ser. B (Engl. Ed.), 38 (2018), pp. $1583-1590$.

[36] — Comparison principles for some fully nonlinear subelleiptic equations on the Heisenberg group, to appear in Analysis in Theory and Applications, (2018).

[37] Y. Y. Li AND L. ZHANG, Liouville-type theorems and Harnack-type inequalities for semilinear elliptic equations, J. Anal. Math., 90 (2003), pp. 27-87.

[38] N. S. Trudinger, Comparison principles and pointwise estimates for viscosity solutions of nonlinear elliptic equations, Rev. Mat. Iberoamericana, 4 (1988), pp. $453-468$. 
[39] J. A. Viaclovsky, Conformal geometry, contact geometry, and the calculus of variations, Duke Math. J., 101 (2000), pp. 283-316.

[40] _ C Conformally invariant Monge-Ampère equations: global solutions, Trans. Amer. Math. Soc., 352 (2000), pp. 4371-4379.

[41] B. Wang, Removable singularities for degenerate elliptic Pucci operator on the Heisenberg group, Nonlinear Anal., 160 (2017), pp. 177-190. 